Mitteilung aus dem Kant. Spitale Basel-Land.

\title{
Hernia obturatoria incarcerata sinistra, Laparotomie, Genesung.
}

Von Chefarzt Dr. L. Gelpke, Dozent für Chirurgie.

Von Brüchen des eirunden Loches sind bis jetzt etwa 200 Fälle beschrieben. Garéngeot, Henry d'Obré, Roman Fischer Thiele, Auerbach, Piqué et Poirier, Englisch, Dehner, Rose, Rörte, Krönlein, Bernhard, Poelchen, Sträter, O. Eix, Sprengel, Schwarzschild, Heuström, Schopf, Grünberg und andere haben je einen oder mehrere Fälle beigetragen. Immerhin bietet diese Bruchform anch heute noch lebhaftes Interesse, vor allen Dingen wegen der ungewöhnlich großen Lebensgefahr des Leidens.

Nach Rose, welcher in der Deutschen Zeitschr. f. Chir. 1893, Bd. 35161 Fälle gesammelt hat, beträgt dieselbe nicht weniger als 78,7 Proz. Von den 161 waren 115 eingeklemmte Brüche des eirunden Loches; davon wurden 12 geheilt durch Taxis, 3 wurden mit Kotfistel entlassen und nur 10 wurden operativ geheilt. Ähnlich gibt Graser eine Mortalität an von 79 Proz.

Es ist demnach dringendes Bedürfnis den Ursachen dieser Bösartigkeit nachzugehen, um die Prognose tunlichst zu bessern.

Aber auch ätiologisch ist die Hernia obturatoria überaus wichtig; merkwürdigerweise befällt sie fast nur Weiber, meist ältere und solche, welche öftere Geburten durchgemacht haben. Zu beiden Beziehungen ist der vorliegende Fall charakteristisch:

Barbara W. von W., Bauersfrau, 72 Jahre alt, groB und mager, hat drei Geburten, dagegen keine schwereren Krankheiten durchgemacht und zeitlebens harte Feldarbeit verrichtet. Seit Jahren leidet sie an 4 Brüchen, als zwei Leisten- und zwei Schenkelbrüchen. Der Hausarzt Dr. Th. in G. stellte die Diagnose auf Hernia obturat. incarcerata auf Grund ansgesprochener Ileuserscheinungen und einer deutlichen, schmerzhaften und druckempfindlichen Resistenz unterhalb des l. horizontalen Schambeinastes. Etwa 20 Stunden nach Auftreten der ersten Schmerzen kam die Frau nach vierstündiger Fahrt durch gebirgige Gegend zur Operation in unsere An- 
stalt. Wir fanden bei der vaginalen Untersuchung eine deutliche schmerzhafte Resistenz im kleinen Becken links, bestätigten im übrigen die Diagnose in allen Teilen und beschlossen im vorliegenden Falle aus später zu erörternden Gründen die Laparotomie auszuführen, statt der sonst allgemein üblichen Herniotomie, wie sie Schmidt, Berger und Graser empfehlen. Bei Beckenhochlagerung gelangte man ohne Mühe an eine unterhalb des Schambeinastes festgehaltene Darmschlinge; (das Débridement) der Entspannungsschnitt wird aus anatomischen Rücksichten nach unten innen ausgeführt, wo voraussichtlich am wenigsten Gefäße zutreffen, der zufühlende Finger kommt hier nicht auf eine pulsierende Ader, während an der oberen äuBeren Peripherie deutlich die Arteria obturatoria zu fühlen ist. Jetzt wird die Darmschlinge schonend aus dem Bruchsacke zurückgezogen und zwar so, daß etwa ausflieBender Darminhalt nicht in die Bauchhöhle gelangen konnte. Die Schlinge erweist sich als nicht geborsten, aber mit zwei brandigen Streifen an der Einklemmungsstelle; dieselben werden nach der von uns seit ungefähr dem Jahre 1894 geübten und seinerzeit im Korrespondenzbl. f. S. A. 1896 veröffentlichten Verfahren quer übernäht ${ }^{1}$ ). Darauf wird der Darm versenkt und mit einigen Seidennähten die Bruchpforte, gleichzeitig mit den darüberliegenden Leisten- und Schenkellücken verschlossen. Die Nachbehandlung bestand in subkutanen und rektalen Salzwassereinläufen. Die Patientin konnte am 10. Tage das Bett verlassen.

Während die Operation einer anderen eingeklemmten Hernie 10-15 Proz. Mortalität gibt, so beträgt diejenige der Einklemmung der Hern. obturat., siehe oben, 75 Proz., und doch ist der Bruch des eirunden Loches ein Bruch wie ein anderer. Der Grund dieser auffallenden Erscheinung liegt in der mangelhaften Diagnose, etwas weniger in der mangelhaften Behandlung. Betreffend die Diagnose schreibt Krönlein (Verh. d.Vers. d. Naturforscher u. Ärzte, Heidelberg 1898): „Die Diagnose der freien Hernia obturatoria scheint kaum möglich, diejenige der eingeklemmten mindestens unsicher; Tatsache ist, daß die meisten Fälle letzterer Kategorie erst auf dem Seziertische erkannt wurden".

Unter den 118 Fällen, welche Englisch zusammengestellt hat, befinden sich 78 eingeklemmte, nicht durch Taxis reponierte Fälle; von diesen 78 Fällen wurden aber nur 18 richtig als Brüche des eirunden Loches erkannt; meist wurde Ileus aus unbekannter Ursache angenommen; sehr häufig wurde eine gleichzeitig bestehende Hernia cruralis resp. inguin. beschuldigt und auch freigelegt, so im Falle von Henry d'Obré, Nußbaum, Hollstein, Hassel-

1) Dieses Übernähen von brandigen Streifen und auch Darmwandbrüchen, auch von Feurer u. A. empfohlen, ist von uns in etwa 8 Fällen immer mit gutem Erfolge ausgeführt worden. 
schwander, Parci, Wilke u. a. In der Regel wurde an Hernia obturatoria überhaupt nicht gedacht, und das ist die Hauptursache der mangelhaften Diagnostik: Die Hernia obturat. ist zu selten, zu wenig bekannt, als daß man mit ihr rechnet; sobald man aber an diese Möglichkeit bei vorliegendem Darmverschluß denkt, sobald man sich erinnert, daß diese Bruchform fast nur bei Frauen und zwar bei älteren vorkommt, welche noch anderweitige Brüche haben, wird man auch meistens imstande sein mit Hilfe der vaginalen und rektalen Untersuchung, mit Hilfe der Abtastung des Scarpaschen Dreieckes, mit Hilfe des Ausschlusses einer anderen Ursache, die Diagnose auf Hernia incarcerata obturator. mit genügender Wahrscheinlichkeit zu stellen. Eine regelwidrige Geschwulst oder Vorwölbung unterhalb des Schambeinastes wird meistens zu entdecken, eine lokale Schmerzhaftigkeit, auf Druck vermehrt, wird immer festzustellen sein; das berühmte Romberg-Howshipsche Symptom: Ausstrahlende Schmerzen bis zum Knie herab, folgend dem Verlaufe des von der Hernie gequetschten Nervus obturatorius, ist auch sehr gut zu verwerten, kommt aber leider nur in der Hälfte der Fälle vor. Ist einmal die Diagnose richtig gestellt, so darf man sich mit der Taxis nicht lange aufhalten. Hat dieselbe schon bei den übrigen eingeklemmten Hernien einen schlechten Leumund wegen der von den praktischen Ärzten immer noch zu wenig gewürdigten Gefahren, der Scheinreduktion, der Verschleppung und der Perforation, so ist bei der Hernia obturator. die Taxis geradezu verboten, denn erstens sind die Patienten fast alle dekrepide Frauen, zweitens steht die versteckte Lage einer schonenden Taxis im Wege, drittens sind die Brüche häufig Darmwandbrüche (nach Englisch fast ein Drittel unter 78 entweder operierten oder obduzierten Fällen 22 mal) oder sehr kleine Brüche, welche rasch brandig wurden; auch die Erleichterung der Taxis durch die Narkose ist im allgemeinen zu verwerfen, da es gefährlich ist, den gleichen Patienten in kurzer Zeit zweimal zu narkotisieren: Zur Taxis und kurz daranf zur Operation.

Auch durch die Veränderung des Operationsverfahrens dürften nach meiner Meinung die Schrecken und Gefahren des Hüftlochbruches gemildert werden.

Bis jetzt wurde allgemein die Herniotomie im Scarpaschen Dreieck mit einer queren Durchschneidung des Kammuskels als Normalverfahren empfohlen (Berger, Schmidt, Graser). Die Laparotomie wurde zwar häufig ausgeführt, aber nicht in der Absicht eine Hernia obturatoria zu operieren, sondern bei irrtüm- 
licher Diagnose. Soviel ich aus der mir zugänglichen Literatur ersehe, ist nur in einem Falle und zwar von Bardenheuer der Bauchschnitt mit Absicht bei richtiger Diagnose ausgeführt worden. Als unsere 72 jährige Patientin, angegriffen von ihrer Krankheit und von der langen Fahrt, bei Nacht hereingebracht wurde, waren wir keinen Augenblick im Zweifel, daß hier der Angriff von der Bauchhöhle aus das schonendere, unblutigere, raschere und sichere Verfahren sei; und ich möchte die Laparotomie bei Hernia obturatoria incarc. unbedingt als das Verfahren der Wahl hinstellen, denn die Furcht vor dem Bauchschnitt war vor 20 und mehr Jahren durchaus am Platze, heute aber nicht mehr; zweitens ist das eirunde Loch von unten viel schwieriger und mit viel größerem Zeitund Blutverlust zu erreichen, als von der Bauchhöhle aus; ein übersichtliches, bequemes Operationsfeld ist überhaupt nicht zu gewinnen, sondern man arbeitet in einem Loch. Bei einer etwaigen Fehldiagnose - ein Fall, mit dem man hier immer rechnen muß - ist die Laparotomie ungleich bequemer, um den Darm abzusuchen. Häufig kam bei der Herniotomie ein Entschlüpfen des Darmes in die Bauchhöhle vor, worauf nachträglich der Bauch geöffnet werden mußte und zwar, soviel ersichtlich, immer mit letalem Ausgang. Ferner muß man bei dem Bruche des Hüftloches mehr als bei jeder anderen Hernie auf Gangrän des Darmes gefaßt sein, das Hervorziehen des Darmes behufs Besorgung der Gangrän ist aber bei der Hermotomie angesichts der starren Wandungen des Canal. obturatorius nach übereinstimmendem Urteil der Autoren überaus schwierig und gefährlich. Schließlich und fünftens ist die Radikaloperation von der Bauchhöhle aus mindestens ebenso zweckmäßig und wirksam auszuführen als von unten. Kurz die Laparotomie hat bei der vorliegenden chirurgischen Aufgabe alle Vorteile für sich und wird allem Anscheine nach in Zukunft das Verfahren die Wahl werden.

Zum Schlusse noch einige Bemerkingen über die Entstehungsursachen der Hernie des eirunden Loches. Dieselben sind überaus interessant. Warum kommt dieser Bruch fast nur bei älteren Franen, nie im Kindesalter, sehr selten beim männlichen Geschlechte vor? Weil das weibliche Becken vermöge seines größeren Querdurchmessers, seiner stärkeren Neigung (wie die meisten Autoren meinen) zur Entstehung der genannten Bruchform disponiert ${ }^{1}$ ). Überaus

1) Nach Englisch (1891) kommen 12 Fälle bei Frauen auf 1 Fall beim Manne vor, seit 1891 habe ich in der Literatur 1 Fall bein Manne (Fall 
häufig kommt die Hernia obturator. mit anderen: Schenkel-, Leisten-, Nabelhernien gleichzeitig vor; 4-5 Brüche an einem und demselben Becken sind nichts seltenes. Auch unsere Patientin zeigt ein solches Bruchbecken - zwei Schenkel-, zwei Leistenbrüche und einen Bruch des Hüftloches. Das beweist, daß die Hernia obturator. eine ausgesprochene Erschlaftungshernie (Hernie de faiblesse) ist, und zweitens, was mir bis jetzt zu wenig gewürdigt scheint, daß die Gewebsveränderung, welche die Schwangerschaft mit sich bringt eine erste Rolle bei der Entstehung der Brüche spielt.

Mit Recht unterscheiden wir zur Zeit zwei große Gruppen von Brüchen, die angeborenen auf einen Bildungsfehler beruhend auch bei kräftigen Menschen häufig vorkommend, dahin gehört eine große, wenn nicht die größere Zahl der äußern Leistenbrüche und der Nabelbrüche. Die zweite Gruppe wird gebildet durch die „Erschlaffungsbrüche", hervorgerufen durch eine abnorme Nachgiebigkeit der Gewebe. Diese regelwidrige Schwäche der Gewebe, vom Verfasser in einer früheren Publikation (Jahresb. d. Kt. Spit. B. L. 1908) Ptoseophilie oder besser Histomalacie genannt, ist ein Konstitutionsfehler. Solche Leute mit ungewöhnlich brüchiger Faser stammen gewöhnlich aus Familien, in welchen Brüche, Vorfall der Gebärmutter und des Mastdarmes, Krampfadern, Lungenblähung, Hängebauch und Senkung der Baucheingeweide, eventuell auch Herzerweiterung erblich sind. Jedem Arzte sind diese Tatsachen geläufig, doch wird es eine verdienstliche Aufgabe der Pathologie sein, das Wesen dieser angeborenen Histomalacie anatomisch festzustellen, ebenso wie die wichtigen Beziehungen zwischen Hernien, Lungenemphysem, Varizen usw. Als Ursache der erworbenen Gewebserschlaffung ist neben Husten, Überanstrengung usw. vor allen Dingen die Schwangerschaft zu nennen. Dafür ist gerade die Hernia obturator. ein sprechendes Beispiel. Die Gravidität bewirkt eine spezifische Auflockerung und Erweichung der Gewebe -- der Knochen sowohl wie der Weichteile wie bekannt teils zum Zweck der Raumgewinnung, teils zum Zweck der direkten Erleichterung der Geburt. Die Erweichung geht von der Gebärmutter als Zentrum aus und hört oberhalb und unterhalb des Beckens allmählich auf und ist eine Analogie der pathologischen Gewebserweichung bei der puerperalen Histo- und Osteomalacie

Bernhardt) auf 14 Fälle bei Frauen gefunden - bekanntlich gibt es auch Männer mit weiblichem Habitus und breitem weiblichen Becken. Unter 16 Jahren fand Englisch keinen Fall; von 16-30 Jahren 7 Fälle. 
264 Gexpke, Hernia obturatora incarcerata sinistra Laparotomie Genesung.

(vgl. auch die physiologische Histo-Osteomalacie in der Schwangerschaft in der Publikation des Verfassers: Beiträge zur Kenntnis der Osteomalacie, Basel, Lonis Jenke 1891). Diese gestative Gewebserweichung des Beckens und seiner Weichteile ist in der Regel nur teilweise rückgängig zu machen. Auf ihre beiläufig überaus dankbare Therapie und Prophylaxe (Orthopädie und Gymnastik der Bauchmuskeln, Liegekur usw.) einzugehen, ist hier nicht unsere Aufgabe. Es genügt darauf hinzuweißen, daß sie die Ursache ist der starken Disposition älterer Frauen zu Schenkelbrüchen und besonders zil Hernia obturatoria. 